Gynecologic and

Obstetric Investigation
Gynecol Obstet Invest 2019;84:313-325

DOI: $10.1159 / 000495347$
Received: May 15, 2018

Accepted after revision: November 11, 2018

Published online: December 14, 2018

\title{
Ulipristal Acetate before Hysteroscopic and Laparoscopic Surgery for Uterine Myomas: Help or Hindrance?
}

\author{
Simone Ferrero ${ }^{a, b}$ Valerio Gaetano Vellone ${ }^{c}$ Fabio Barra ${ }^{a, b}$ Carolina Scala ${ }^{a, b}$ \\ ${ }^{a}$ Academic Unit of Obstetrics and Gynecology, IRCCS Ospedale Policlinico San Martino, Genoa, Italy; ${ }^{\text {b Department }}$ \\ of Neurosciences, Rehabilitation, Ophthalmology, Genetics, Maternal and Child Health (DiNOGMI), University of \\ Genoa, Genoa, Italy; ${ }^{\mathrm{C}}$ Department of Surgical and Diagnostic Sciences, IRCCS Ospedale Policlinico San Martino, \\ Genoa, Italy
}

\section{Keywords}

Hysteroscopy · Laparoscopy · Myoma · Myomectomy ·

Ulipristal acetate

\begin{abstract}
Background/aims: Ulipristal acetate ([UPA], Esmya $\left.{ }^{\circledR}\right)$ is an orally active selective progesterone-receptor modulator that has been approved as preoperative treatment for uterine myomas. This systematic review aims to summarize the available data on surgical outcomes of patients undergoing myomectomy by hysteroscopy or by laparoscopy after preoperative treatment with UPA. Methods: this review was performed following PRISMA guidelines and was registered in PROSPERO (CRD42018092201). PubMed, EMBASE, and Medline databases were systematically searched electronically until March 2018 for keywords concerning this topic. There were included only peer-reviewed, English language journal articles. Results: two prospective studies and 4 retrospective studies were included. The effect of UPA on the endometrium does not increase the technical difficulty during hysteroscopic myomectomy, but it increases the chance of complete primary resection in complex hysteroscopic myomectomies. Moreover, UPA does not increase the overall technical difficulty of laparoscopic myomectomy. Contradic-
\end{abstract}

tory results have been reported on the impact of UPA on the separation of the myoma from the myometrium and on the consistency of the myomas. Conclusion: current results support the use of UPA prior to both surgical procedures. Further studies should confirm these preliminary findings and to assess the long-term outcomes of myomectomies following UPA treatment.

(c) 2018 S. Karger AG, Basel

\section{Introduction}

Uterine myomas occur in $20-40 \%$ of women of reproductive age [1]. Although a large number of factors may be involved in the development and growth of uterine leiomyomas, the specific pathogenesis of these benign tumors remains unknown [2]. The majority of patients with myomas are asymptomatic; anyway, some women may have symptoms and signs of varying degrees, which sometimes severely affect quality of life. The treatment options for uterine myomas include expectant management, medical therapy, and surgical options [3].

Among medical therapy, ulipristal acetate ([UPA], known also as CDB-2914, Esmya ${ }^{\circledR}$ ), is an emerging op-

\section{KARGER}

(c) 2018 S. Karger AG, Basel

E-Mail karger@karger.com

www.karger.com/goi
Simone Ferrero, $\mathrm{MD}, \mathrm{PhD}$

Academic Unit of Obstetrics and Gynecology

IRCCS Ospedale Policlinico San Martino

Largo R. Benzi 10, IT-16132 Genoa (Italy)

E-Mail simone.ferrero@ unige.it 
tion to preoperatively treat myomas. This drug is a selective progesterone-receptor modulator (SPRM) that binds with high affinity to progesterone receptor exerting both antagonist and partial agonist activity. The final activity as an agonist or antagonist is due to the interaction with nuclear coactivators and corepressors within specific cell types that influences the expression of progesterone-related genes. The UPA administration increases apoptosis of myoma cells by enhancing cleaved caspase- 3 and by decreasing B-cell lymphoma-2 transcriptions [4]. Moreover, it causes change of the expression of proangiogenic proteins, such as vascular endothelial growth factor or adrenomedullin [5], and reduces the amount of extracellular matrix by decreasing production of collagen and enhancing that of metalloproteinases $[6,7]$. Furthermore, inducing amenorrhea without downregulating levels of estradiol, UPA does not cause estrogen deprivation-related symptoms, differently from gonadotropin releasing hormone analogs (GnRH-a) [8]. Because of its pharmacokinetic characteristics [9], UPA can be taken orally once daily.

Two short-term double-blind randomized phase III trials (PEARL I and II) demonstrated the efficacy of UPA (3-months therapy) in controlling uterine bleeding as well as decreasing uterine myoma size. Importantly, these trials reported positive data on the safety profile of this SPRM, which was well tolerated by the majority of patients treated $[10,11]$. Furthermore, the PEARL II trial showed that UPA was noninferior in obtaining control of uterine bleeding and caused a lower incidence of adverse effects (AEs) in comparison to GnRH-a (leuprolide acetate). However, UPA failed to demonstrate a higher decrease in uterine volume [11]. Subsequently, the double-blind randomized PEARL III trial demonstrated that the long-term intermittent therapy with UPA (1-4 treatment cycles of 3 months each) caused a high rate of amenorrhea, improved patients' quality of life, and was safe [12]. Recently, another large prospective multicenter descriptive study including 1,473 women confirmed UPA efficacy and safety when administrated preoperatively for treating moderateto-severe symptoms related to uterine myomas [13].

Surprisingly, despite the UPA approval in Europe and Canada as preoperative treatment of uterine myomas and the large number of treated patients in last these years, little attention has been given to the outcomes of patients undergoing surgery after this hormonal treatment. This systematic review aims to summarize the available data on surgical outcomes of patients treated for uterine myomas by hysteroscopy or by laparoscopy after receiving treatment with UPA.

\section{Methods}

This systematic review was performed according to the PRISMA guidelines [14] and was registered in the PROSPERO register (www.crd.york.ac.uk/PROSPERO CRD42018092201). The search was conducted in accordance with the principles outlined in the Cochrane Handbook for Systematic Reviews of Interventions [15]. All authors participated in the design of the search strategy and of the inclusion and exclusion criteria. No institutional review board approval was needed because only published, deidentified data were analyzed.

\section{Search Strategy and Process}

PubMed, EMBASE, and Medline databases were systematically searched electronically from inception until March 2018 (last research $30 \mathrm{March}$, 2018). Combination of the following relevant medical subject heading (MeSH) terms, keywords, and word variants were used: "UPA" alone or in combination with "myomas," "fibroid," "laparoscopy," "hysteroscopy," "hysterectomy," "myomectomy," and "surgery." All pertinent articles were carefully assessed, and their reference lists were evaluated to identify any other study that could be included in this review.

\section{Inclusion and Exclusion Criteria}

In this systematic review, only peer-reviewed, English language journal articles concerning the preoperative administration of UPA for uterine myomas were included. In particular, the following topics were covered: short and long-term clinical and surgical efficacy of UPA administration before hysteroscopic myomectomy and laparoscopic myomectomy. Randomized controlled trials (RCTs), prospective controlled studies, prospective cohort studies or retrospective studies, reviews, case series, and case reports were considered eligible. Case reports and small case series ( $<10$ cases) were evaluated only if they contained highly valuable information. Letters to the editor and abstracts accepted at conferences were excluded from the review.

\section{Data Extraction}

Two authors (S.F., C.S.) performed the study selection by a 2 -stage process. The first step was to assess eligibility based on the titles and abstracts. In the second step, the decision for final inclusion was made after evaluating carefully the full papers and their references. The authors were not blinded to the names of the investigators or the sources of publication. Disagreements generated in the process of study selection and data collection were resolved by consensus among all 4 authors (S.F., V.G.V., F.B., C.S.).

\section{Quality Assessment}

Two authors (V.G.V., F.B.) independently evaluated the methodological quality of the study groups included in this systematic review. Quality assessment of the nonrandomized cohort and case-control study included in this review was performed using the Newcastle-Ottawa Scale (NOS) [16]. According to the NOS, each study is judged based on 3 broad perspectives: the selection of the study groups, the comparability of the groups, and the ascertainment of the outcome of interest. Assessment of the comparability of the study groups includes the evaluation of the representativeness of the exposed cohort, the selection of the nonexposed cohort, the ascertainment of exposure, and the demonstration that 
Fig. 1. PRISMA flow-chart.

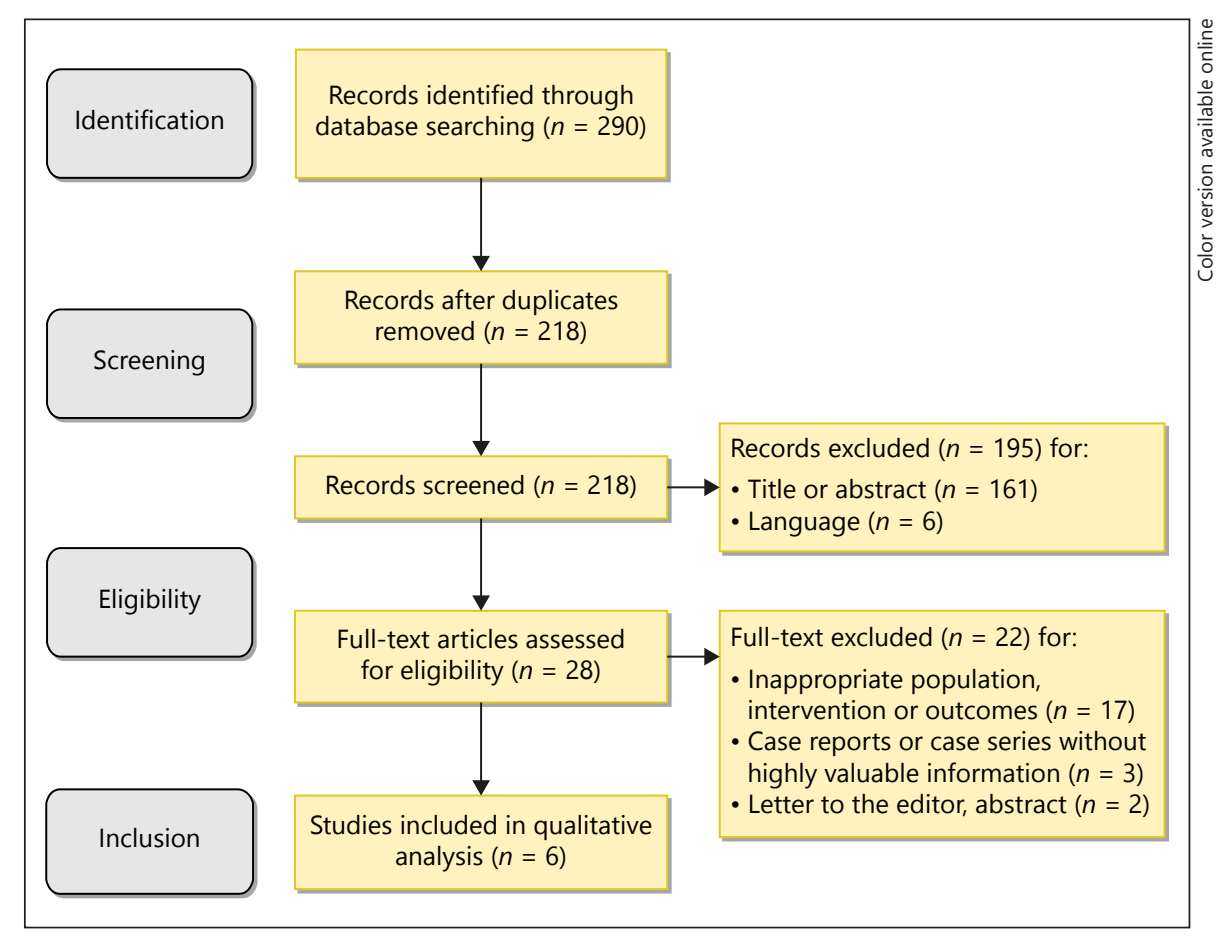

the outcome of interest was not present at the start of the study. Assessment of the comparability of the study includes the evaluation of the comparability of cohorts based on the design or analysis. Finally, ascertainment of the outcome of interest includes the evaluation of the type of the assessment of the outcome of interest, length and adequacy of the follow-up. According to NOS, a study is awarded a maximum of one star for each numbered item within the selection and outcome categories. A maximum of 2 stars is given for comparability.

\section{Results}

\section{Study Selection and Study Characteristics}

The literature search (Fig. 1), based on our predefined key search items, identified 218 publications after the removal of duplicates. The titles of these publications were screened and assessed for eligibility, and, finally, 28 fulltext articles were evaluated to be definitively included in the systematic review. Among these, 22 studies were excluded for the inappropriate population, intervention or outcomes $(n=17)$, and because they were case reports or series without highly valuable information $(n=3)$, letter to the editor or abstract $(n=2)$. In the end, 6 studies $(2$ prospective studies $[17,18]$ and 4 retrospective studies [19-22]) were included in the present review. Two studies evaluated UPA use before hysteroscopic myomectomy $[17,19,20], 2$ studies evaluated it before laparoscopic myomectomy [21, 22], and 1 study before both surgical techniques [18]. Given the characteristics of the studies included and the heterogeneity of the available articles in terms of methodology, no meta-analysis was attempted. The quality of the study included in the review is shown in the online supplementary Table 1 (see www.karger. com/doi/10.1159/000495347).

\section{UPA Prior to Hysteroscopy}

When UPA is administered before hysteroscopic myomectomy, it has potential advantages and disadvantages. First, it causes a decrease in myoma volume, thus theoretically facilitating the myomectomy. Furthermore, amenorrhea is rapidly induced avoiding irregular bleeding, facilitating the scheduling of surgery. On the other hand, it is well known that UPA may increase endometrial thickness and be responsible for the appearance of Progesterone Receptor Modulator Associated Endometrial Changes [8]. It is debated whether these endometrial changes may negatively affect the intrauterine visualization of the myomas, thus being disadvantageous when performing the hysteroscopic myomectomy.

Four studies included in this review investigated the use of UPA before hysteroscopic myomectomy (Table 1). The first study was an Italian single-center prospective nonrandomized comparative pilot trial [17]. It compared 


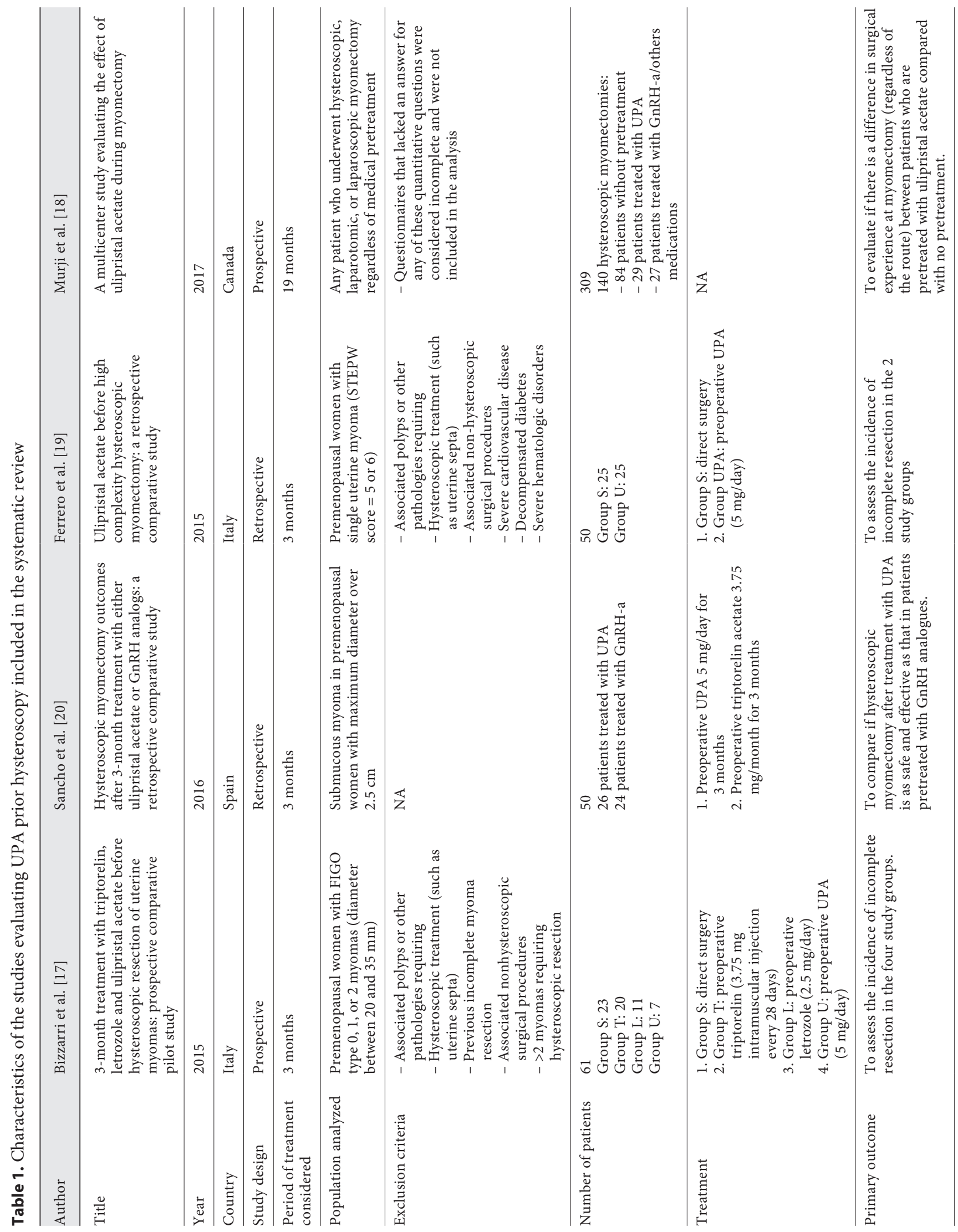




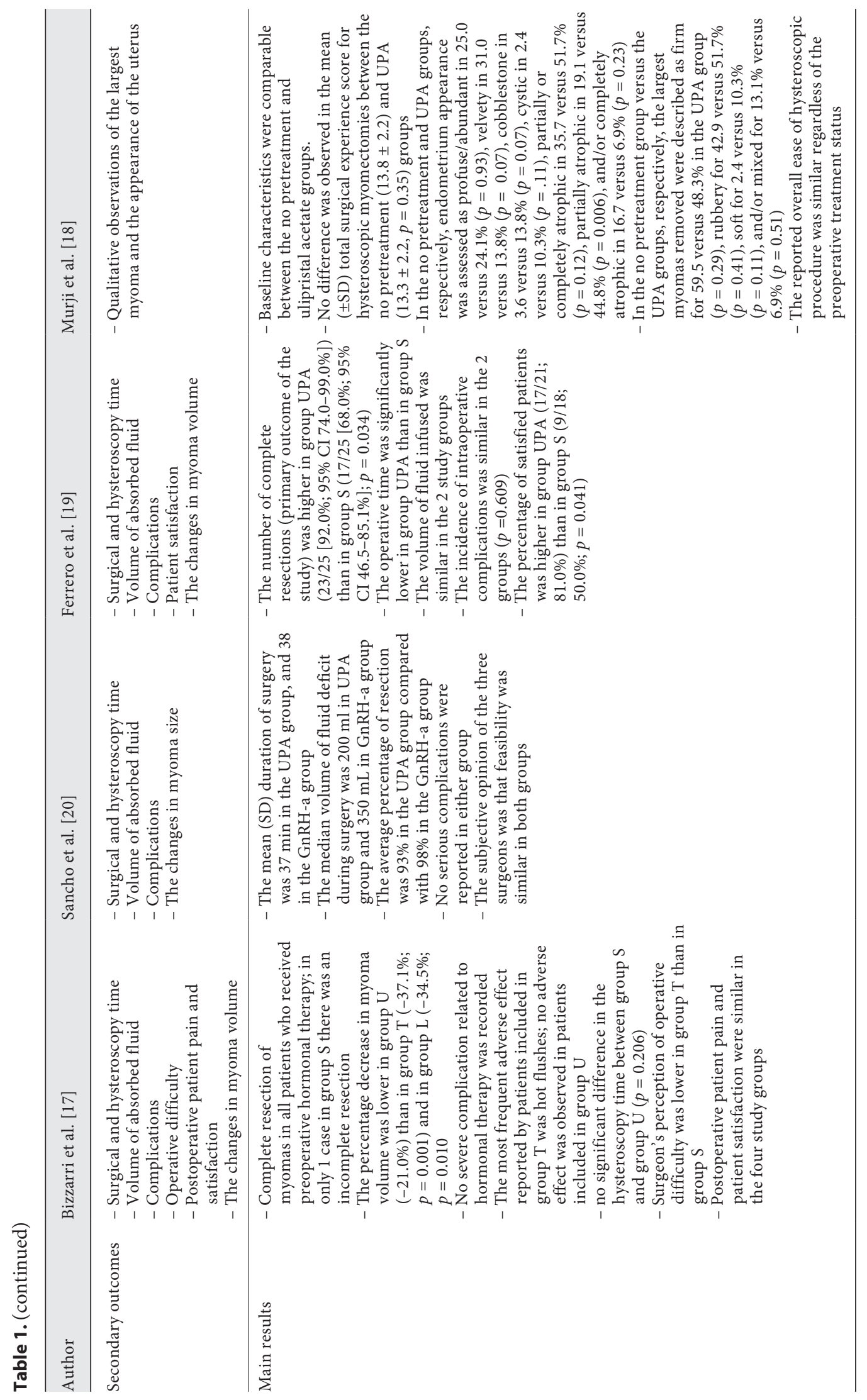


the efficacy of different hormonal therapies and the absence of treatment prior to perform the hysteroscopic removal of type 0,1 , or 2 myomas (with diameter ranging from 20 to $35 \mathrm{~mm}$ ), according to The International Federation of Gynecology and Obstetrics (FIGO) classification [23]; in this study, the myomas were also classified according to the size, topography, extension, penetration, wall (STEPW) classification [24, 25]. A total of 61 women underwent either direct surgery $(n=23)$ or received a 3 -month preoperative therapy with triptorelin (a GnRHa, $3.75 \mathrm{mg}$ intra-muscular injection every 28 days; $n=20$ ), letrozole (an aromatase inhibitor, $2.5 \mathrm{mg} /$ day; $n=11$ ), or UPA ( $5 \mathrm{mg}$ oral daily; $n=7$ ). There was not significantly difference in type of the myomas, according to the FIGO and STEPW classifications among the 4 study arms. All hormonal therapies succeeded in causing a significant decrease in the largest myoma volume, even if there was a significantly lower decrease of volume in patients treated with UPA. Moreover, all women who received these medical treatments had a complete resection of myomas, with no significant differences between the groups. On contrary, 1 patient $(4.3 \%)$ who did not receive any preoperative drug had an incomplete resection of myomas. During the hysteroscopic myomectomy, there was no case of uterine perforation or fluid overload. Furthermore, patients who received triptorelin and letrozole had better operative results (shorted length of surgery, lower volume of fluid infused, and lower fluid absorption) in comparison with patients who did not receive any preoperative treatment. A limitation of the study is represented by the fact that surgeons were not blinded to the use of UPA, triptorelin, or letrozole. Anyway, the surgeon's perception of operative difficulty (rated on a 10-point visual analog scale) was lower in patients treated with triptorelin than in those not receiving any preoperative treatment. Postoperative pain and patient satisfaction were similar in the 4 study groups. Because of the small number of patients treated with UPA, this study did not allow to draw conclusion of the usefulness of this preoperative treatment. Although patients treated with UPA had higher endometrial thickness at surgery (mean $6.9 \mathrm{~mm}$ ) than those treated with other drugs, the characteristics of the endometrium were not perceived as a difficulty during the resection by the surgeons.

Sancho et al. [20] retrospectively evaluated hysteroscopic resections of submucosal myomas with largest diameter $>2.5 \mathrm{~cm}$ after 3 months treatment with UPA ( $5 \mathrm{mg}$ daily; $n=26)$ in comparison to triptorelin acetate $(3.75$ $\mathrm{mg}$ intramuscular injection every 28 days; $n=24)$. The myomas were classified accordingly to the European So- ciety for Gynecological Endoscopy classification [26]. A higher rate of patients with type 2 myomas were present in the UPA arm. In the UPA arm, all the hysteroscopic myomectomies were performed after the last dose of the medical treatment (between 10 and 20 days) and before the first menstruation. Most of the women (92\%) were surgically treated by using bipolar resectoscope with saline solution. This study demonstrated that there was no difference in myoma size before undergoing surgery in both arms. Moreover, the mean length of surgery ( $37 \mathrm{~min}$ in the UPA group and $38 \mathrm{~min}$ in the $\mathrm{GnRH}$-a group) and the median volume of fluid deficit $(200 \mathrm{~mL}$ in UPA group and $350 \mathrm{~mL}$ in $\mathrm{GnRH}$-a group) were similar in the 2 study arms. The surgeons subjectively estimated the percentage of myoma resection during hysteroscopy by comparing the initial volume and the residual myoma at the end of the surgery; the percentage of resection was $93 \%$ in the UPA arm and 98\% in the GnRH-a arm. Finally, the subjective opinion of the feasibility for the surgical procedures reported by the surgeons was similar for both drugs: in particular, cervix dilatation was slightly easier in the UPA group, whereas visualization of the uterine cavity was slightly clearer in the GnRH-a group. Anyway, these variables were considered to be of little or no clinical importance.

Another Italian retrospective trial evaluated the efficacy of 3-month preoperative treatment with UPA in women undergoing high complexity hysteroscopic myomectomy (for STEPW score 5 or 6 myomas) [19]. Fifty patients either underwent direct surgery or received a 3-month preoperative treatment with UPA. The choice of treatment was based on patient preference. The surgeons were aware of the use of preoperative treatment. During the 3-month treatment with UPA, the largest myoma obtained a mean percentage volume decrease of $21.9 \%$. The number of complete resections (primary outcome) was significantly higher in patients treated with UPA (92.0\%) than in those receiving no treatment (68.0\%). Moreover, the operative time was significantly lower in women treated with UPA; in contrast, similar volumes of fluid infused and fluid balance were demonstrated in both study groups. Although the incidence of intraoperative complications was similar in the 2 groups, this trial was underpowered for detecting differences in the incidence of rare events (such as uterine perforation). In patients who received UPA, the surgeons rated the additional difficulty to the procedure caused by the thickened endometrium by using a 3-point Likert scale (much worse, worse, and neutral). No patient included in the study had endometrial thickness $>16 \mathrm{~mm}$ at the time of surgery; the sur- 
geons judged that the thickening of the endometrium added an increase in the difficulty of the surgical procedure only in 4 women (16.0\%), whereas there was no negative influence in the majority of them (84.0\%).

A recent prospective observational multicenter study evaluated the UPA impact on hysteroscopic myomectomy by using a web-based questionnaire [18]. Among 140 hysteroscopic myomectomies included in this trial, $60 \%$ were performed without preoperative hormonal therapy, $21 \%$ after UPA treatment, and 19\% after other hormonal therapies (leuprolide acetate alone, leuprolide acetate plus UPA, oral contraceptive pills, or progestins). No difference was reported in the surgical experience for performing hysteroscopic myomectomies between patients not receiving preoperative treatment and those receiving UPA. In addition, there is no difference regarding quality of hysteroscopic visualization between these 2 groups. Furthermore, qualitative assessments of endometrial appearance obtained similar rates of profuse/abundant endometrium regardless of the group. A not negligible limitation of this study was that the surgeons were not blinded to the patients' clinical history. Furthermore, over $80 \%$ of the women enrolled had the largest myomas of FIGO type 0 or 1 , which are likely to be more easily resected without the need of receiving a preoperative treatment.

Currently, an on-going phase IV RCT (NCT02361879) is comparing UPA ( $5 \mathrm{mg}$ /day for 3 months) and leuprolide acetate (11.25 mg intra-muscular injection in the luteal phase) before undergoing hysteroscopic resection of submucosal myomas. The primary objective of this study is to compare the proportion of controlled uterine bleeding in patients preoperatively treated by UPA or by the GnRH-a. The secondary objectives of this study are to evaluate the feasibility of the myoma hysteroscopic resection, the operative time, the hemoglobin concentration (the day before and $3 \mathrm{~h}$ after the hysteroscopy), and the fluid absorption during surgery in the 2 study groups.

It is known that a potential effect of UPA is to change the position of the myoma within the uterine wall. In particular, this phenomenon (named "myoma migration") has also been described after the administration of other preoperative hormonal therapies, such as GnRH-a [2729], and other procedures, such as uterine artery embolization. Because of myoma migration, some authors reported cases of submucosal myomas scheduled for hysteroscopic resection that, after UPA treatment and migration, required laparoscopic myomectomy [30], had myoma expulsion and required a vaginal myomectomy $[30,31]$, or required no further treatment [30].

UPA before Hysteroscopic and Laparoscopic Myomectomy

\section{UPA Prior to Laparoscopy}

The major concern of laparoscopic surgeons is that the preoperative treatment with UPA may complicate myomectomy because of a change in the consistency of the myomas, which are presumed to become softer after this hormonal treatment. Considering the relevance of the traction maneuvers during laparoscopic myomectomy, the difficulty in adequately grasping the benign tumor may be a critical element in influencing the operative time. Furthermore, a decreased consistency of the myomas may theoretically increase the difficulty in differentiating these lesions from the surrounding endometrium/ myometrium. Thus, the enucleation of the myomas may become more challenging.

Despite the wide administration of UPA to patients with uterine myomas and the extensive use of laparoscopic surgery for this setting, only 3 studies reported the surgical outcomes of patients submitted to laparoscopic myomectomy (Table 2).

A retrospective study based on a prospectively collected database assessed the usefulness of 3-month preoperative treatment with UPA prior to laparoscopic excision of large uterine myomas in women suffering heavy menstrual bleeding [21]. A total of 86 patients included in the study had a main myoma (FIGO type 3, 4, or 5) with diameter $\geq 10 \mathrm{~cm}$ and had of up to 3 myomas. The main diameters of the other myomas (FIGO type 3, 4,5 , or 6 ) were $\leq 5 \mathrm{~cm}$ (for the second myoma) and $\leq 3$ $\mathrm{cm}$ (for the third myoma). Patients underwent either direct surgery $(n=43)$ or received 3 -month preoperative therapy with UPA $(n=43)$. The UPA treatment obtained a $31.8 \%$ and a $13.5 \%$ decrease in the volume and the main diameter of the largest myoma. In particular, patients who received UPA had at surgery lower diameter and volume of the largest myoma, lower total myomas volume, and higher hemoglobin levels in comparison with patients who did not receive any preoperative treatment. No patient required conversion to laparotomy. Moreover, the operative time was shorter in patients treated with UPA, and also the intraoperative blood loss and the hemoglobin drop were significantly lower in this group of patients. Six patients (14\%) who underwent direct surgery and no patient in the UPA group required a postoperative blood transfusion. A potential limitation of this study is that no intraoperative technique was employed to decrease the blood loss (such as vasopressin injection in the area surrounding the myoma or the intravenous injection of tranexamic acid). The incidence of complications was similar between the 2 study groups. 


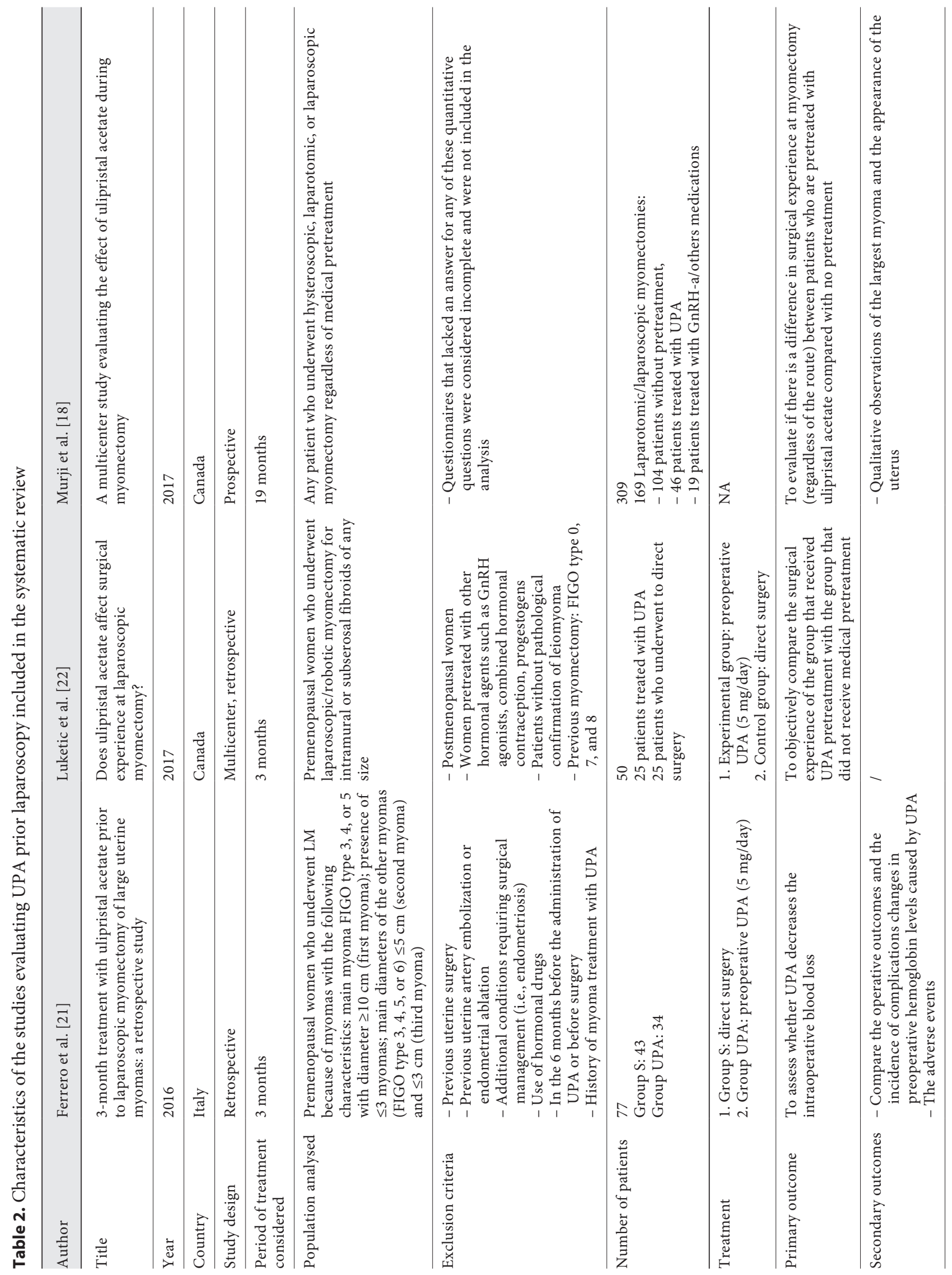




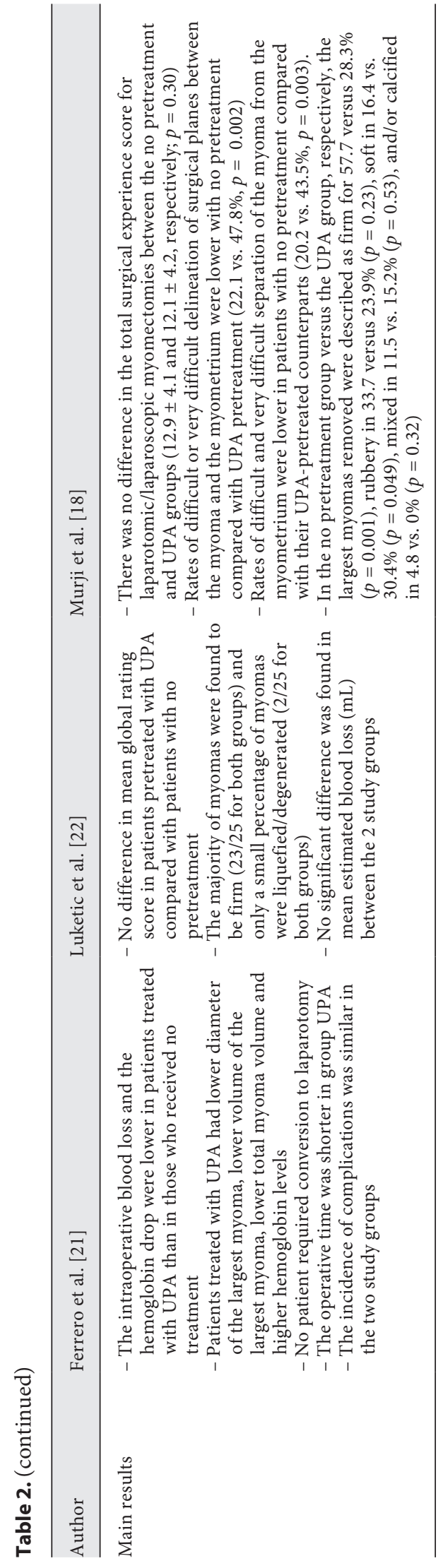

UPA before Hysteroscopic and Laparoscopic Myomectomy
More recently, a retrospective, multicenter cohort study evaluated the surgical experience of laparoscopic/ robotic myomectomy for patients pretreated with UPA $(n=25)$ in comparison with patients untreated $(n=25)$ [22]. This study was based on a review of videos of laparoscopic/robotic myomectomies that was blindly performed by 2 physicians by using a standardized rating tool. The study demonstrated that there was no difference between the 2 groups regarding the depth of the myometrial incision. Moreover, the ease of the myoma-myometrium cleavage plane identification and the myoma detachment was similar in both groups. At least, there was no difference in blood loss during and after myoma detachment as well as in their consistency between the 2 groups. Regarding myoma consistency, the author demonstrated that the majority of myomas were firm (23/25 for both groups) and only a small percentage of them were liquefied/degenerated ( $2 / 25$ for both groups). Therefore, the authors concluded that UPA does not seem to have a major impact on the consistency of uterine myomas and on the difficulty encountered by the surgeons in laparoscopic/robotic enucleation. In particular, even if all surgeons periodically encountered difficult cases in which the planes between the myoma and the myometrium were adherent and the bleeding was above average, the authors stated that these difficulties might have not been attributable to the preoperative treatment with UPA but rather to the myoma or uterine anatomy itself. Furthermore, the authors reported that UPA did not impact negatively the intraoperative blood loss [22]; however, this observation based only on a retrospective chart review may have been inaccurate in estimating the hemoglobin drop caused by surgery [32].

A recent prospective observational multicenter study evaluated the UPA efficacy on laparotomic or laparoscopic myomectomy by performing a web-based questionnaire [18]. There was no difference in the surgical experience for patients who received in comparison to those who did not receive any preoperative therapy. However, in contrast with the findings of previous trials, the authors stated that the delineation of surgical planes as well as the separation of the myomas from the myometrium was more difficult after UPA pretreatment. Moreover, myomas were also described as being softer in patients receiving it.

Two ongoing RCTs are assessing the preoperative administration of UPA in patients undergoing laparoscopic myomectomy. One RCT (NCT02361905) is including women with cellular myomas, which are more often soft and appear more tanned or yellow and less circumscribed 
than the usual leiomyomas. It has been demonstrated that patients with hypoechoic myomas have significantly longer surgery time after treatment with GnRH-a than patients with other types of myomas or than patients untreated with hypoechoic myomas [33]. Interestingly, the greater softening of the myomas is probably related to degenerative changes induced by the preoperative treatment with GnRH-a. At histology, these myomas have a predominance of areas of coagulative necrosis as well as myxoid degeneration. Theoretically, cellular myomas may be more responsive to UPA administration than common myomas, given its antiproliferative activity, which induces apoptotic necrosis. Moreover, in comparison to the GnRH-a-induced myxoid necrosis, which get worse the surgical procedure on the tumor, UPA probably allows an easier enucleation. This ongoing RCT enrolling women with cellular myomas is evaluating a preoperative treatment with UPA in comparison to leuprolide acetate. The primary outcome of this study is to estimate the difference in operative times between the 2 study arms. The secondary outcomes are the evaluation of the difficulty of leiomyoma enucleation perceived by surgeon on a VAS scale, the estimation of the intraoperative patients' blood loss as well as the assessment of postoperative pathological characteristics of myomas. Another multicenter double-blind RCT (named MYOMEX; NCT02288130) is investigating if UPA is noninferior to leuprolide acetate in terms of intraoperative blood loss (primary outcome), surgical time, surgical ease, complications, quality of life, and costs. In the inclusion criteria of this study, there are patients with a maximum of 2 FIGO type $3,4,5,6$, or 2-5 myomas with diameter between 5 and $12 \mathrm{~cm}$ (maximum diameter); in patients eligible, other fibroids should be small $(<2 \mathrm{~cm})$, not clinically relevant, or not resectable (e.g., for difficult position), or type 7 (any size).

\section{Discussion}

In the last years, preoperative treatment for myomas, such as $\mathrm{GnRH}-\mathrm{a}$, progestins, dopamine agonists, prostaglandin analogs, selective estrogen receptor modulators, and SPRM have been deeply investigated. The choice of which drug should be eventually prescribed before surgery should take into account the characteristic of myomas (size, number, and location), efficacy of treatment, incidence and severity of AEs, route of administration, preference of patients and surgeons [34]. Anyway, all these preoperative medical options share some potential drawbacks such as cost, delay in the surgical procedure, additional consultations, and drug-related AEs.

$\mathrm{GnRH}-\mathrm{a}$ has been routinely employed in this setting, as they are able to increase hemoglobin levels before surgery (reducing uterine bleeding), to decrease uterine and myomas size, and to reduce blood loss and the need for blood transfusion. However, during the treatment with these drugs, women are more likely to experience estrogen deprivation-related AEs.

UPA can be preoperatively considered in women that are eligible for receiving any type of surgery for myomas, since it confers similar clinical advantages while awaiting surgery, considerably improving patients' quality of life [34]. A better safety profile has always been considered the most important advantage of this drug compared with other hormonal therapies (such as $\mathrm{GnRH}-\mathrm{a}$ and aromatase inhibitors). Although the most common AEs experienced with UPA administration are headache, breast tenderness, and hot flushes, the majority of them have a mild-to-moderate intensity and do not usually lead to the discontinuation of treatment [9]. However, recently, 4 cases of serious liver injury (3 of which ended in liver transplantation) have been reported in women under treatment with UPA. Therefore, after an accurate investigation, the Pharmacovigilance Risk Assessment Committee of the European Medicines Agency has concluded that UPA may have contributed to the development of liver injury, and, for this reason, currently recommends to put in place several measures in order to minimize the risk of these rare but serious AEs [35].

Recently, a systematic review by Cochrane have analyzed the data available in the literature on the preoperative medical therapy for myomas: when compared with $\mathrm{UPA}, \mathrm{GnRH}-\mathrm{a}$ is associated with a greater reduction in uterine volume but is more likely to cause AEs, such as hot flushes. Moreover, there is no a clear difference in bleeding reduction or hemoglobin levels among the 2 hormonal options [36].

Some studies described in our systematic review showed that by shrinking the myomas UPA may facilitate minimally invasive surgical procedures (such as laparoscopic or hysteroscopic myomectomy). Theoretically, the decrease in myoma volume due to the use of a preoperative drug may also allow performing less invasive surgical procedures; for example, in the case of large uteri requiring morcellation for laparoscopic or vaginal removal, the administration of this drug may decrease uterine size thus avoiding the need to perform an eventual morcellation [37]. However, up to now, this potential benefit for UPA has not been yet demonstrated in published studies. 
A recent systematic review and meta-analysis reported that UPA has not been sufficiently evaluated yet for surgical outcomes [38]. Similarly, there are only limited data on the comparison between preoperative SPRMs and other hormonal therapies in patients undergoing hysteroscopic or laparoscopic myomectomy.

In general, direct hysteroscopic myomectomy can usually be considered in women with single FIGO type 0 or 1 myomas without a necessity to prescribe any preoperative treatment [17]. The preoperative administration of UPA for myomas should be considered in the case of complex surgical procedures because it offers the advantage of a higher chance of complete primary resection without increasing the difficulty of surgery [19]. In women of reproductive age, operative hysteroscopy should be done when the endometrium is as thin as possible, as this makes intrauterine procedures easier, reduces operative time, and the severity of bleeding. Several medical treatments (such as vaginal danazol, oral contraceptives, and single progestins, GnRH-a) have been tested by several authors to achieve an optimal endouterine view due to the reduced endometrial thickness and intraoperative bleeding. Nevertheless, to date no definitive data allow a conclusion about the best preoperative medical management to employ for preparing endometrium before hysteroscopy surgery. However, hormonal treatment before hysteroscopic surgery in reproductive-age women is not mandatory, since it is possible to obtain moderate endometrial hypotrophy performing the surgical procedure immediately after the menstruation [39-41].

Several trials showed that the effect of UPA on the endometrium does not increase the technical difficulty during hysteroscopic myomectomy [17-20]. Anyway, it is still debated whether the Progesterone Receptor Modulator Associated Endometrial Changes may negatively affect the intrauterine visualization of myomas when performing hysteroscopic myomectomy [8]. Moreover, further studies with larger sample size to assess the impact of UPA on the incidence of rare surgical complications (such as uterine perforation) lack in the literature.

Direct surgery for myomas should be performed in patients that have sufficient hemoglobin levels; if the patient is anemic, preoperative menstrual suppression and iron supplementation should be suggested to improve the outcomes of surgery. Anyway, it is important to under light that laparoscopic myomectomy can be complicated by extensive peri-operative bleeding; therefore, blood transfusions and eventually reoperation may be required in some patients, especially if with previous low hemoglobin levels. Regarding this topic, there is evidence that UPA decreases the uterine bleeding and increases the preoperative hemoglobin level of patients with uterine myomas; furthermore, it also decreases intraoperative blood loss and, therefore, the risk of a subsequent transfusion. Obviously, a higher postoperative hemoglobin levels enable a quicker return to social activities including work.

Based on the studies included in this review, UPA does not also seem to increase the overall technical difficulty of laparoscopic myomectomy $[18,21,22]$. However, contradictory results have been reported on its impact on the separation of the myoma from the myometrium and on the consistency of the myomas after the treatment [18, $22]$. Further studies should be done to confirm these preliminary findings as well as to evaluate long-term outcomes (such as frequency of myoma recurrence, recovery time, and quality of life) of laparoscopic myomectomy following UPA treatment.

Prior to prescribing UPA, particularly in women with abnormal uterine bleeding, the characteristics of myomas and of uterine cavity should always be assessed by performing an accurate ultrasonography. The increase in myoma size despite the use of UPA may be suggestive of the presence of a uterine sarcoma [13, 42, 43]. Furthermore, it had recently been described a case of well-differentiated endometrial cancer diagnosed 5 months after 2 courses (3-month each) of therapy with UPA [44].

Up to date, few studies on surgical outcomes after UPA administration are present in the literature. In the near future, new randomized prospective studies with larger sample size are needed to confirm these positive preliminary results. Importantly, it should be performed by an accurate comparative evaluation between preoperative UPA and GnRH-a. Additionally, among studies, more homogenous end-points should be established in order to allow future accurate meta-analysis on this topic. New studies are also required in order to assess the long-term outcomes of laparoscopic or hysteroscopic myomectomies (such as frequency of myoma recurrence, recovery time, and quality of life). Moreover, future research should focus on cost-effectiveness and distinguish between groups of women with fibroids who would most benefit.

\section{Disclosure Statement}

The authors have nothing to disclose.

\section{Funding Source}

The paper was not funded.
UPA before Hysteroscopic and Laparoscopic Myomectomy
Gynecol Obstet Invest 2019;84:313-325 DOI: $10.1159 / 000495347$ 


\section{References}

1 Parker WH: Etiology, symptomatology, and diagnosis of uterine myomas. Fertil Steril 2007;87:725-736

2 Laganà AS, Vergara D, Favilli A, La Rosa VL, Tinelli A, Gerli S, Noventa M, Vitagliano A, Triolo O, Rapisarda AM, Vitale SG: Epigenetic and genetic landscape of uterine leiomyomas: a current view over a common gynecological disease. Arch Gynecol Obstet 2017; 296:855-867.

3 Parker WH: Uterine myomas: management. Fertil Steril 2007;88:255-271.

4 Xu Q, Takekida S, Ohara N, Chen W, SitrukWare R, Johansson ED, Maruo T: Progesterone receptor modulator CDB-2914 downregulates proliferative cell nuclear antigen and $\mathrm{Bcl}-2$ protein expression and up-regulates caspase- 3 and poly(adenosine $5^{\prime}$-diphosphate-ribose) polymerase expression in cultured human uterine leiomyoma cells. J Clin Endocrinol Metab 2005;90:953-961.

5 Xu Q, Ohara N, Chen W, Liu J, Sasaki H, Morikawa A, Sitruk-Ware R, Johansson ED, Maruo T: Progesterone receptor modulator CDB-2914 down-regulates vascular endothelial growth factor, adrenomedullin and their receptors and modulates progesterone receptor content in cultured human uterine leiomyoma cells. Hum Reprod 2006;21:24082416.

$6 \mathrm{Xu}$ Q, Ohara N, Liu J, Amano M, Sitruk-Ware R, Yoshida S, Maruo T: Progesterone receptor modulator CDB-2914 induces extracellular matrix metalloproteinase inducer in cultured human uterine leiomyoma cells. Mol Hum Reprod 2008;14:181-191.

7 Cox J, Malik M, Britten J, Lewis T, Catherino WH: Ulipristal acetate and extracellular matrix production in human leiomyomas in vivo: a laboratory analysis of a randomized placebo controlled trial. Reprod Sci 2018;25: 198-206.

8 Tafi E, Scala C, Leone Roberti Maggiore U, Bizzarri N, Candiani M, Venturini PL, Ferrero S: Drug safety evaluation of ulipristal acetate in the treatment of uterine fibroids. Expert Opin Drug Saf 2015;14:965-977.

9 Ferrero S, Vellone VG, Barra F: Pharmacokinetic drug evaluation of ulipristal acetate for the treatment of uterine fibroids. Expert Opin Drug Metab Toxicol 2018;14:107-116.

10 Donnez J, Tatarchuk TF, Bouchard P, Puscasiu L, Zakharenko NF, Ivanova T, Ugocsai G, Mara M, Jilla MP, Bestel E, Terrill P, Osterloh I, Loumaye E; PEARL I Study Group: Ulipristal acetate versus placebo for fibroid treatment before surgery. N Engl J Med 2012;366: 409-420.

11 Donnez J, Tomaszewski J, Vazquez F, Bouchard P, Lemieszczuk B, Baro F, Nouri K, Selvaggi L, Sodowski K, Bestel E, Terrill P, Osterloh I, Loumaye E; PEARL II Study Group: Ulipristal acetate versus leuprolide acetate for uterine fibroids. N Engl J Med 2012;366:421432 .
12 Donnez J, Vázquez F, Tomaszewski J, Nouri K, Bouchard P, Fauser BC, Barlow DH, Palacios S, Donnez O, Bestel E, Osterloh I, Loumaye E; PEARL III and PEARL III Extension Study Group: Long-term treatment of uterine fibroids with ulipristal acetate. Fertil Steril 2014;101:1565-1573 e1561-1518.

13 Fernandez H, Schmidt T, Powell M, Costa AP Arriagada P, Thaler C: Real world data of 1473 patients treated with ulipristal acetate for uterine fibroids: Premya study results. Eur J Obstet Gynecol Reprod Biol 2017;208:9196.

14 Moher D, Liberati A, Tetzlaff J, Altman DG; PRISMA Group: Preferred reporting items for systematic reviews and meta-analyses: the PRISMA statement. Int J Surg 2010;8:336341.

15 collaboration TC: Cochrane Handbook for Systematic Reviews of Interventions version 5.0.2, 2011.

16 Wells G, Shea B, O’Connell D, Peterson J, Welch V, Losos M, Tugwell P: The Newcastle-Ottawa Scale (NOS) for assessing the quality of nonrandomised studies in meta-analyses. http://www. ohri.ca/programsclinical_epidemiology/ oxford.asp (accessed August 18, 2018).

17 Bizzarri N, Ghirardi V, Remorgida V, Venturini PL, Ferrero S: Three-month treatment with triptorelin, letrozole and ulipristal acetate before hysteroscopic resection of uterine myomas: prospective comparative pilot study. Eur J Obstet Gynecol Reprod Biol 2015; 192:22-26.

18 Murji A, Wais M, Lee S, Pham A, Tai M, Liu G: A multicenter study evaluating the effect of ulipristal acetate during myomectomy. J Minim Invasive Gynecol 2018;25:514-521.

19 Ferrero S, Racca A, Tafi E, Alessandri F, Venturini PL, Leone Roberti Maggiore U: Ulipristal acetate before high complexity hysteroscopic myomectomy: a retrospective comparative study. J Minim Invasive Gynecol 2016; 23:390-395.

20 Sancho JM, Delgado VS, Valero MJ, Soteras MG, Amate VP, Carrascosa AA: Hysteroscopic myomectomy outcomes after 3-month treatment with either Ulipristal Acetate or $\mathrm{GnRH}$ analogues: a retrospective comparative study. Eur J Obstet Gynecol Reprod Biol 2016;198:127-130.

21 Ferrero S, Alessandri F, Vellone VG, Venturini PL, Leone Roberti Maggiore U: Threemonth treatment with ulipristal acetate prior to laparoscopic myomectomy of large uterine myomas: a retrospective study. Eur J Obstet Gynecol Reprod Biol 2016;205:4347.

22 Luketic L, Shirreff L, Kives S, Liu G, El Sugy R, Leyland N, Solnik MJ, Murji A: Does ulipristal acetate affect surgical experience at laparoscopic myomectomy? J Minim Invasive Gynecol 2017;24:797-802.

23 Munro MG, Critchley HO, Fraser IS; FIGO Menstrual Disorders Working Group: The
FIGO classification of causes of abnormal uterine bleeding in the reproductive years. Fertil Steril 2011;95:2204-2208, 2208 e2201e2203.

24 Lasmar RB, Barrozo PR, Dias R, Oliveira MA: Submucous myomas: a new presurgical classification to evaluate the viability of hysteroscopic surgical treatment - preliminary report. J Minim Invasive Gynecol 2005;12:308311 .

25 Lasmar RB, Xinmei Z, Indman PD, Celeste RK, Di Spiezio Sardo A: Feasibility of a new system of classification of submucous myomas: a multicenter study. Fertil Steril 2011;95: 2073-2077.

26 Wamsteker K, Emanuel MH, de Kruif JH: Transcervical hysteroscopic resection of submucous fibroids for abnormal uterine bleeding: results regarding the degree of intramural extension. Obstet Gynecol 1993;82:736740 .

27 Wen L, Tseng JY, Wang PH: Vaginal expulsion of a submucosal myoma during treatment with long-acting gonadotropin-releasing hormone agonist. Taiwan J Obstet Gynecol 2006;45:173-175.

28 Yu KJ, Lai CR, Sheu MH: Spontaneous expulsion of a uterine submucosal leiomyoma after administration of a gonadotropin-releasing hormone agonist. Eur J Obstet Gynecol Reprod Biol 2001;96:223-225.

29 Kriplani A, Agarwal N, Parul D, Bhatla N, Saxena AK: Prolapsed leiomyoma with severe haemorrhage after GnRH analogue therapy. J Obstet Gynaecol 2002;22:449-451.

30 Willame A, Marci R, Petignat P, Dubuisson J: Myoma migration: an unexpected "effect" with Ulipristal acetate treatment. Eur Rev Med Pharmacol Sci 2016;20:1439-1444.

31 Chantraine F, Poismans G, Nwachuku J, Bestel E, Nisolle M: Expulsion of a uterine myoma in a patient treated with ulipristal acetate. Clin Case Rep 2015;3:240-242.

32 Ferrero S, Scala C, Barra F: Regarding "Does Ulipristal Acetate Affect Surgical Experience at Laparoscopic Myomectomy?”. J Minim Invasive Gynecol 2018;25:541.

33 Zullo F, Pellicano M, Di Carlo C, De Stefano R, Marconi D, Zupi E: Ultrasonographic prediction of the efficacy of GnRH agonist therapy before laparoscopic myomectomy. J Am Assoc Gynecol Laparosc 1998;5:361-366.

34 Singh SS, Belland L, Leyland N, von Riedemann S, Murji A: The past, present, and future of selective progesterone receptor modulators in the management of uterine fibroids. Am J Obstet Gynecol 2018;218: 563-572.e1.

35 Agency EM: Esmya: New Measures to Minimise Risk of Rare but Serious Liver Injury, 2018.

36 Lethaby A, Puscasiu L, Vollenhoven B: Preoperative medical therapy before surgery for uterine fibroids. Cochrane Database Syst Rev 2017;11:CD000547. 
37 Vitale SG, Sapia F, Rapisarda AMC, Valenti G, Santangelo F, Rossetti D, Chiofalo B, Sarpietro G, La Rosa VL, Triolo O, Noventa M, Gizzo S, Lagana AS: Hysteroscopic morcellation of submucous myomas: a systematic review. Biomed Res Int 2017;2017:6848250.

38 de Milliano I, Twisk M, Ket JC, Huirne JA, Hehenkamp WJ: Pre-treatment with GnRHa or ulipristal acetate prior to laparoscopic and laparotomic myomectomy: a systematic review and meta-analysis. PLoS One 2017;12:e186158.

39 Lagana AS, Giacobbe V, Triolo O, Granese R, Ban Frangez H, Vrtacnik-Bokal E, Ietto C, Palmara VI: Dienogest as preoperative treatment of submucous myomas for hysteroscop- ic surgery: a prospective, randomized study. Gynecol Endocrinol 2016;32:408-411.

40 Lagana AS, Palmara V, Granese R, Ciancimino L, Chiofalo B, Triolo O: Desogestrel versus danazol as preoperative treatment for hysteroscopic surgery: a prospective, randomized evaluation. Gynecol Endocrinol 2014;30: 794-797.

41 Lagana AS, Vitale SG, Muscia V, Rossetti P, Buscema M, Triolo O, Rapisarda AM, Giunta L, Palmara V, Granese R, Frangez HB, Romano A: Endometrial preparation with Dienogest before hysteroscopic surgery: a systematic review. Arch Gynecol Obstet 2017;295: 661-667.
42 Kadhel P, Smail M, Borja De Mozota D: Inefficiency of ulipristal acetate on uterus leiomyomas as an additional sign to suspect leiomyosarcoma. J Gynecol Obstet Hum Reprod 2017;46:609-611.

43 Istre O: Unexpected uterine leiomyosarcoma during laparoscopic hysterectomy treated 6 months with ulipristal acetate and contained power morcellation. J Minim Invasive Gynecol 2017;24:198.

44 Ignatov T, Eggemann H, Dan Costa S, Ignatov A: Endometrial cancer after ulipristal acetate for uterine fibroma. Eur J Obstet Gynecol Reprod Biol 2017;219:134. 